\title{
Parasitic-Free Modulation of Semiconductor Lasers
}

\author{
KERRY J. VAHALA, MEMBER, IEEE, AND MICHAEL A. NEWKIRK
}

\begin{abstract}
Active-layer photomixing is a technique for modulating semiconductor lasers with nearly perfect immunity to device parasitics. We have previously demonstrated this technique at room temperature [2]. In this paper, we measure the intrinsic modulation response of a laser diode using this technique at temperatures as low as 4.2 K. From these measurements, the temperature dependence of important dynamical parameters is determined. In addition, this provides a stringent test of the active-layer photomixing technique since parasitic response is degraded, while the intrinsic response is improved for low-temperature operation. At $4.2 \mathrm{~K}$, the ideal intrinsic response is measured for frequencies as high as $15 \mathrm{GHz}$ despite an estimated parasitic corner frequency of $410 \mathrm{MHz}$.
\end{abstract}

\section{INTRODUCTION}

$\mathrm{T}$ HE frequency response of a semiconductor laser to injection current modulation is a superposition of two independent response functions: the parasitic response and the intrinsic response. The parasitic response results from package and chip-related impedances that tend to shunt modulation current around the active layer of the device and thus inhibit efficient modulation of the laser diode beyond some corner frequency. The intrinsic response is determined by the interaction dynamics of the lasing mode and the gain medium. Both response functions must be considered when a semiconductor laser is to be optimized for application in a high-data-rate optical communication system. In most cases, the parasitic response is determined by the $R C$ corner frequency associated with the chip's contact-layer resistance and the contact-layer capacitance [1]. Thus, any reduction in either of these quantities (e.g., better contacts to reduce the resistance or the use of semiinsulating substrates to reduce the capacitance) leads to significant improvements in the parasitic response. The intrinsic response is more fundamental. It not only sets an upper limit on modulation response, assuming parasitics can be reduced, but also gives information on the basic physics of the device and on the material system in which the device is fabricated [1]. Measurement of the intrinsic or fundamental response is therefore of both practical and fundamental importance.

The separation of the intrinsic response function from the overall device response is difficult, especially in stateof-the-art high-speed structures that are capable of intrinsic response corner frequencies exceeding $10 \mathrm{GHz}$. Pre-

Manuscript received October 4, 1988; revised January 12, 1989. This work was supported in part by the National Science Foundation, in part by the Powell Foundation, in part by the ATT Corporation, and in part by the IBM Corporation.

The authors are with the Department of Applied Physics, California Institute of Technology, Pasadena, CA 91125.

IEEE Log Number 8927325. viously, however, we introduced a new technique called active-layer photomixing that can modulate a semiconductor laser with nearly perfect immunity to parasitic effects at all frequencies [2]-[4]. We demonstrated the technique on a GaAs (AlGaAs) transverse junction stripe (TJS) laser diode, showing that the resulting modulation response is indeed the theoretical ideal. (In a later publication, $\mathrm{Su}$ and co-workers also demonstrated this approach [5].) In this paper, we will present additional results on laser modulation by active-layer photomixing at both liquid nitrogen and liquid helium temperatures. In addition, for completeness, we will review a theoretical explanation of the parasitic-free nature of the active-layer photomixing technique based on an equivalent circuit model [4]. Our purpose in measuring the modulation response at cryogenic temperatures is twofold. First, certain important dynamic parameters can be measured, and their temperature dependence can be correlated to test theoretical explanations. Second, the parasitic response of a laser diode is degraded at low temperature because of carrier freeze-out induced increases in the contact-layer resistance. Simultaneously, however, the intrinsic response is improved, as explained later. Thus, low-temperature operation provides a severe test of the active-layer photomixing modulation technique. In the next section, we will briefly review the basic features of the intrinsic response function and then show that active-layer photomixing is immune to parasitic effects. The latter will make use of an equivalent circuit model introduced in [4]. In Section III, experimental results will be presented.

\section{Active-Layer Photomixing}

In active-layer photomixing, the outputs from two single-frequency lasers (one tunable) are superimposed onto the active layer of a laser diode (see Fig. 1). By selecting the photon energies of the sources to fall within the bandgap energy of the laser's cladding layers, but well above the bandgap energy of the active layer, the two beams are selectively absorbed in the active layer. The absorption process results in mixing of the fields, thereby generating a population of electrons and holes whose densities are modulated at the difference frequency of the sources. These carriers quickly relax to the bottom of their respective bands, where they contribute to lasing action by modulating the optical gain and hence the laser diode's light output. By controlling the frequency difference of the two sources, the laser diode's light output can be modulated over a wide range of frequencies. In the experiment, the laser is biased to an operating point using an injection 


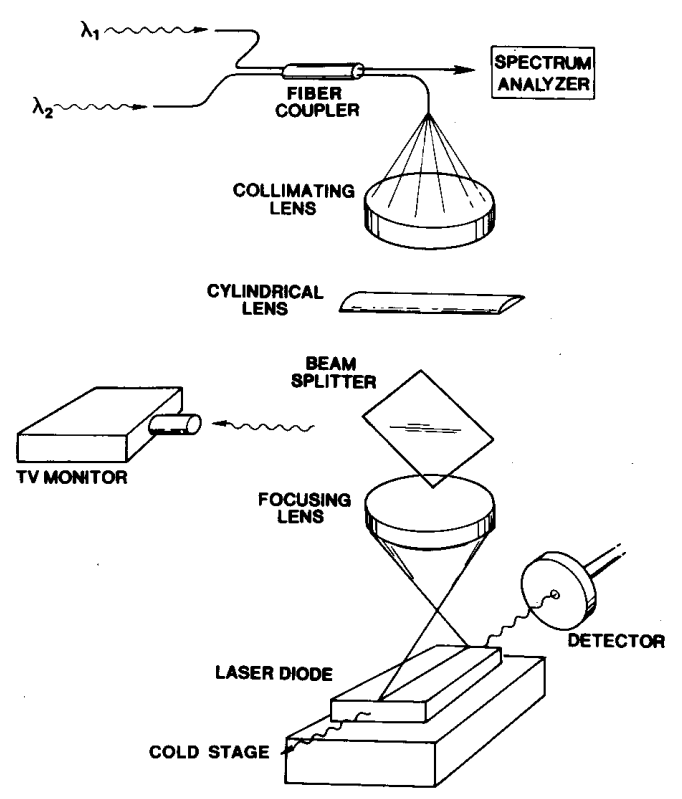

Fig. 1. Diagram of active-layer photomixing experimental setup.

current, and the single-frequency sources are used to small-signal modulate the laser about this point.

In this section, we will show that the modulation produced by active-layer photomixing reflects only the intrinsic dynamics of the laser diode. To do this, we will first review what is meant by the intrinsic frequency response and then compare active-layer photomixing to conventional current modulation based on an equivalent circuit model.

\section{A. The Intrinsic Response}

In this section, we will review what is meant by the intrinsic modulation response of a laser diode. We begin by considering the single-mode rate equations for photon density $p$ and carrier density $n$ :

$$
\begin{aligned}
& \dot{p}=\Gamma g(n, p) p-\frac{p}{\tau}+\theta \\
& \dot{n}=-g(n, p) p-r(n)+\frac{I_{L}}{q V}
\end{aligned}
$$

where $\Gamma$ is the fill factor, $g(n, p)$ is the optical gain, $\tau$ is the photon lifetime, $\theta$ is the spontaneous emission rate per unit volume into the optical mode, $r(n)$ is the spontaneous recombination rate per unit volume for the carriers, $I_{L}$ is the current in amps into the intrinsic device, $q$ is the electronic charge, and $V$ is the active-layer volume. These equations are linearized by introducing steady-state and small-signal quantities (e.g., $n=n_{o}+n_{m}$ where $n_{o}$ is the steady-state and $n_{m}$ is the time-varying component) and by Taylor expanding $g(n, p)$ and $r(n)$ at the operating point. Harmonically time-varying modulation is then assumed, and transfer functions relating the small-signal in- jection current amplitude $\hat{I}_{L}$ to the small-signal photon density amplitude $\hat{p}_{m}$ and carrier density amplitude $\hat{n}_{m}$ can then be found. They are given by

$$
\begin{aligned}
\hat{p}_{m} & =\frac{\hat{I}_{L}}{q V} \frac{\Gamma g_{n} P_{o}}{\omega_{R}^{2}-\omega^{2}+i \omega \gamma} \\
\hat{n}_{m} & =\frac{\hat{I}_{L}}{q V} \frac{i \omega+\beta}{\omega_{R}^{2}-\omega^{2}+i \omega \gamma} \\
\beta & =\frac{\theta}{P_{o}}-\Gamma g_{p} P_{o} \\
\omega_{R}^{2} & =\frac{g_{n} P_{o}}{\tau} \\
\gamma & =\gamma_{o}+\tau \omega_{R}^{2}\left(1+\frac{\Gamma\left|g_{p}\right|}{g_{n}}\right)
\end{aligned}
$$

where, for example, $g_{n}$ is the derivative of $g(n, p)$ evaluated at the operating point. It should also be noted that $\gamma_{o}$ is, in general, a constant containing damping contributions from spontaneous emission, diffusion, and other sources. We have lumped these effects into the definition of $\gamma_{o}$, although they are not explicitly included in the rate equations employed here. For a more detailed discussion of these effects, see [6].

Since $\hat{p}_{m}$ is proportional to the output power modulation of the laser, (2a) is the intrinsic response function. It has the overall appearance of a second-order low-pass network. There are three important features of this response function. First, it has a high-frequency rolloff of 20 $\mathrm{dB} /$ decade in output power. This translates into a 40 $\mathrm{dB} /$ decade rolloff in detected power from a photodetector. Second, it exhibits a well-defined resonance near the frequency $\omega_{R}$. From (2d), the square of this frequency varies linearly with output power. Third, the width of the resonance is determined by a damping parameter $\gamma$ that is a linear function of $\omega_{R}^{2}$. The slope of this line is determined by the photon lifetime $\tau$, the differential gain $g_{n}$, and the nonlinear gain parameter $g_{p}$ [7]. We will use these three features as a test of ideality of the measured modulation response curves.

\section{B. Equivalent Circuit Model}

The modulation created by the active-layer photomixing technique does not rely upon electronic transport processes into the device's active layer. Modulation is created where it is needed by the photomixed optical beams. Nonetheless, the modulation is loaded somewhat by device parasitic elements, and it is important to determine the significance of this loading. It will be shown that such loading is, in fact, minute. The clamping of the optical gain above threshold will be seen to cause a decoupling of the intrinsic laser diode response from the external parasitic circuit as viewed by the mixed optical sources.

The laser diode, including parasitic elements, is modeled using the small-signal equivalent circuit shown in 
Fig. 2. The principal parasitic elements included are the bond wire inductance $L$, the contact resistance $R$, the capacitance associated with the chip itself $C$, and the depletion-layer capacitance $C_{D}$. The impedance $Z(\omega)$ represents the small-signal impedance of the intrinsic laser diode, described in greater detail below. The current source, $I_{M}$, results from the carriers created by the photomixing process. These carriers are created high in their respective energy bands and relax on femtosecond time scales to the bottom of the band. The relaxation process results in a small shift in the local quasi-electrochemical potentials or, equivalently, a charging of the depletionlayer capacitance. For efficient active-layer photomixing, the modulation current $I_{L}$ must be large in comparison to the possible parasitic currents $I_{D}$ and $I_{R}$. Typical values for the parasitic elements are $L=1 \mathrm{nH}, R=10 \Omega, C=$ $10 \mathrm{pF}$ ( $1 \mathrm{pF}$ for devices on semiinsulating substrates), and $C_{D}=1-50 \mathrm{pF}$ (depending on structure and threshold level excitation). The equivalent small-signal circuit model for conventional current modulation is shown in Fig. 3. For conventional current modulation, the response associated with the parallel $R C$ circuit normally determines the parasitic response function corner frequency [1].

The laser diode intrinsic impedance $Z(\omega)$ can be determined by using results from the previous section, as illustrated in the analysis of [4]. We reproduce the essentials of that analysis here for convenience. The concept of an impedance for the intrinsic laser diode has been introduced by several groups [8]-[10]. Considering Fig. 2, the small-signal carrier density amplitude can be related to the small-signal voltage across $Z(\omega)$ through the following expression [4], [10]:

$$
\hat{V}_{m}=\frac{M k_{B} T}{q} \frac{\hat{n}_{m}}{n_{o}}
$$

where

$$
M=2+\frac{n_{o}}{2 \sqrt{2}}\left(\frac{1}{N_{t}}+\frac{1}{N_{c}}\right)
$$

Combining (3a) and (2b) allows the intrinsic device impedance $Z(\omega)$ to be identified:

$$
Z(\omega)=\frac{M k_{B} T}{q^{2} V n_{o}} \frac{i \omega+\beta}{\omega_{R}^{2}-\omega^{2}+i \omega \gamma} .
$$

$|Z(\omega)|$ is plotted in Fig. 4 at several photon densities corresponding to laser output powers of $0.5,1.0,5.0$, and $10.0 \mathrm{~mW} /$ facet in a GaAs (AlGaAs) device $250 \mu \mathrm{m}$ in length with uncoated facets (these parameters describe the device measured in [2]). The values for various parameters used in this calculation are given in Table $I$. The values for $g_{p}$ and $r_{n}$ have been determined from the experimental plot of $\gamma$ versus $\omega_{R}^{2}$ appearing in [2]. Notice that the overall magnitude of the intrinsic impedance is quite small. The smallness of this impedance combined with the location of the optical induced current source (as compared to the location of the current source in conventional current modulation) renders the photomix induced mod-

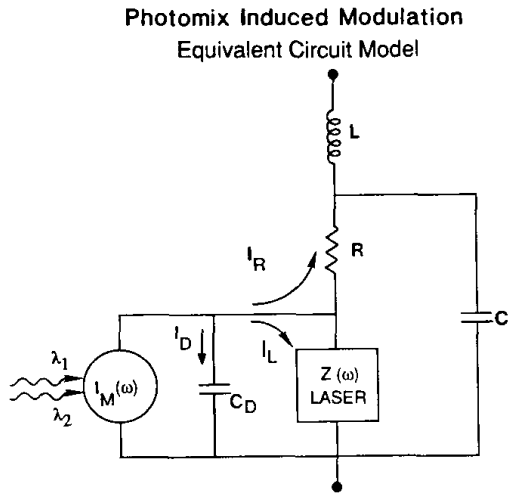

Fig. 2. Equivalent circuit model for active-layer photomixing. The photomix induced current $I_{M}(\omega)$ is divided among three paths associated with the depletion-layer capacitance, the intrinsic laser, and the contact resistance. The components $I_{D}$ and $I_{R}$ are small in comparison to $I_{L}$ at all frequencies.

$$
\text { Convential Current Modulation }
$$

Equivalent Circuit Model

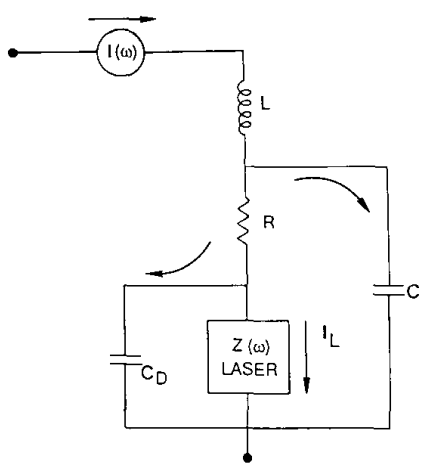

Fig. 3. Equivalent circuit model for conventional injection current modulation. The principal source of parasitic loading is shunting of the injection current $I(\omega)$ through the contact-layer capacitance $C$.

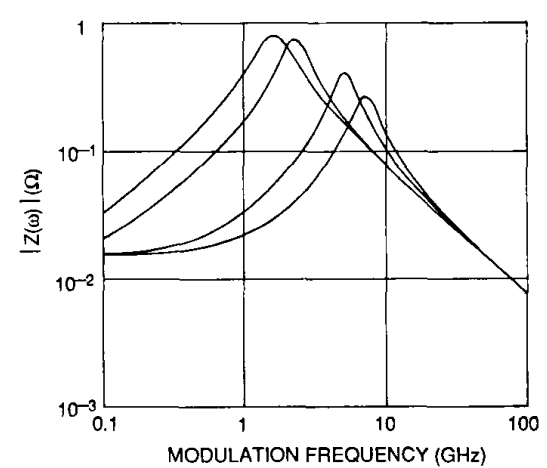

Fig. 4. The intrinsic impedance of the laser diode at output powers per facet of $0.5,1.0,5.0$, and $10.0 \mathrm{~mW}$ (note that the resonant frequency increases with power).

ulation immune to loading, given in terms of $I_{R}$ and $I_{D}$ [4]. As an aside, note that the reverse situation, in which a large $I_{R}$ is desirable, occurs in detectors. For a more detailed discussion, the reader is referred to [4]. 
TABLE I

Laser Parameters Used in the Intrinsic Impedance Calculation

$$
\begin{array}{ll}
V=5.0 \times 10^{-11} \mathrm{~cm}^{3} & r_{n}=5.3 \times 10^{\circ} \mathrm{sec}^{-1} \\
g_{n}=2.5 \times 10^{-6} \mathrm{~cm}^{3} \mathrm{sec}^{-1} & T=3 p s e c \\
g_{p}=-6.9 \times 10^{-6} \mathrm{~cm}^{3} \mathrm{sec}^{-1} & n_{0}=3 \times 10^{18} \mathrm{~cm}^{-\mathrm{s}} \\
\Gamma=0.4 & N_{v}=8.2 \times 10^{18} \mathrm{~cm}^{-5} \\
\theta=7 \times 10^{21} \mathrm{~cm}^{-3} \mathrm{sec}^{-1} & N_{c}=4.3 \times 10^{17} \mathrm{~cm}^{-\mathrm{s}}
\end{array}
$$

In summary, active-layer photomixing produces modulation with nearly perfect immunity to device parasitics for two reasons. First, the modulation is conveyed by optical means into the active layer, i.e., carrier transpot processes are not involved. Second, the modulation, once produced, is effectively decoupled from extrinsic circuit elements by the exceedingly small intrinsic: iripedance of the active layer. It is worth noting that the irt:insic irnpedance is small because of the tendency of the gain (and hence voltage across the intrinsic impedance) to be clamped at its threshold value. Complete ciarnping is never in effect, however. In particular, the nonlinear gain effect is the dominant source of unclamping in the present case and leads to the small but finite low-frequency impedance appearing in Fig. 4.

\section{EXPERIMENTAL Results}

In the experiment, light from a krypton laser and a dye laser are combined as illustrated in Fig. 1 to generate microwave modulation in the semiconductor laser. The details of the low-temperature experiment are essentially the same as those of the room temperature experiment described in [2]. Two important differences should be noted, however. The first is that the krypton laser was operated at a wavelength of $676.4 \mathrm{~nm}$, and the dye laser was adjusted for tunability near this wavelength by employing DCM dye. The room temperature measurement described in [2] used pump wavelengths near $752.5 \mathrm{~nm}$. The change in pump wavelength was necessitated by the bandgap shift of $\mathrm{GaAs}$ as the temperature was reduced. The second change from the room temperature setup was the addition of a low-temperature cryostat. The laser diode resided in the cryostat under vacuum conditions for all measurements. Access to the surface of the laser for pumping and to one of the facets for output light collection was attained through two quartz windows on the cryostat.

The device used in this experiment is a Mitsubishi GaAs TJS laser diode similar to the device employed in the room temperature measurement. This particular device has a transparent contact to facilitate the photomixing process. By "transparent," we mean that the gold contact layer is absent above the active layer, thereby allowing efficient optical coupling. In the TJS laser diode, this opening is a necessary part of the device structure, making it a particularly convenient structure to study using this technique. In structures not having this feature, it is possible to cre- ate orenings in the device contact by removing a small an 3 int of gold at a point along the active layer stripe. We have, in fact, used this approach to apply the active-layer photomixing process to buried heterostructure laser diodes. In addition, active-layer photomixing can also be implemented by facet pumping.

The operational temperatures tested are 293,77 , and 4.2 K. Several interesting things happen as a laser diode is cooled. First, the bandgap energy shifts to larger values. This, as mentioned earlier, necessitates a pump wavelength for which $\mathrm{GaAs}$ strongly absorbs at liquid helium temperature, but for which the $\mathrm{AlGaAs}$ cladding remains transparent at room temperature. Second, carrier freeze-out causes the parasitic contact-layer resistance to increase. This increase is visible in Fig. 5, where the measured laser voltage-current characteristics are displayed. The differential resistance above threshold is seen to increase from $7 \Omega$ at $293 \mathrm{~K}$ to 17 and $39 \Omega$ at 77 and $4.2 \mathrm{~K}$ respectively. This means that the parasitic corner frequency for this device at liquid helium temperature is $410 \mathrm{MHz}$, assuming a $10 \mathrm{pF}$ contact-layer capacitance. Third, the optical gain spectrum narrows as the Fermi occupancy for electrons and holes becomes a sharper function of energy. This has two desirable consequences: the lasing threshold is reduced, and the differential gain is increased. The decrease of laser threshold is evident in the light output versus current curves shown in Fig. 6. It can be seen that the threshold is dramatically reduced to as low as $0.6 \mathrm{~mA}$ at $4.2 \mathrm{~K}$. The larger differential gain for low-temperature operation increases the intrinsic resonance frequency of the laser diode. Thus, for the purpose of testing the active-layer photomixing technique, low-temperature operation is ideal since it adversely affects the laser diode's parasitic response while improving its intrinsic response. In addition, the increase in the differential gain $g_{n}$ will affect the dependence of $\gamma$ on $\omega_{R}^{2}$ [eq. (2e)].

Typical modulation response data are presented in Fig. 7 at the three operating temperatures. The laser diode bias current was adjusted to produce approximately $2 \mathrm{~mW}$ output power per facet at each temperature. The resonance is clearly visible, and there is no indication of any parasitic effects. This is true even for operation at $4.2 \mathrm{~K}$, where, as estimated earlier, the parasitic corner frequency is 410 MHz. The corner frequency of these curves can be seen to increase with decreasing temperature. This increase is primarily due to the increase of the differential gain $g_{n}$. This variation is also apparent in Fig. 8, where we have plotted $\nu_{R}^{2}$ versus output power at the three operating temperatures. The curves again exhibit the linear behavior predicted by theory. From the slope, we estimate that $g_{n}$ increases by $\mathrm{a}$ factor of 7.0 at $77 \mathrm{~K}$ and 13.6 at $4.2 \mathrm{~K}$ over its room temperature value, assuming that the photon lifetime and the modal volume do not change significantly with temperature.

Finally, using the modulation response data, we have plotted $\gamma$ versus $\omega_{R}^{2}$ for each temperature (see Fig. 9). The curves are linear. In addition, the slope is observed to 


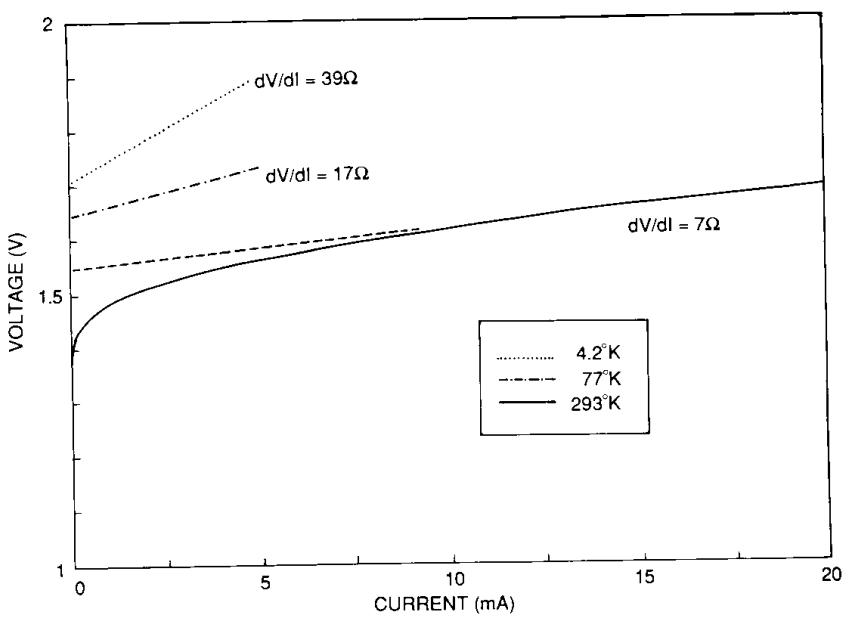

Fig. 5. Laser diode forward-bias voltage-current characteristics at the three operating temperatures. Note that the differential resistance increases from $7 \Omega$ at room temperature to $39 \Omega$ at liquid helium temperature.

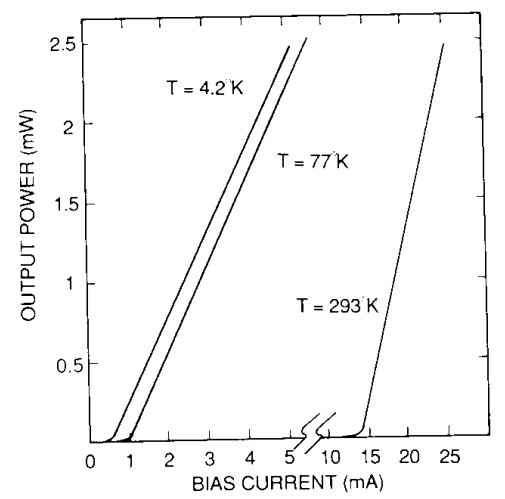

Fig. 6. Lasing output power per facet versus injection current at the three operating temperatures. The threshold current is reduced to $0.6 \mathrm{~mA}$ for liquid helium temperature operation.

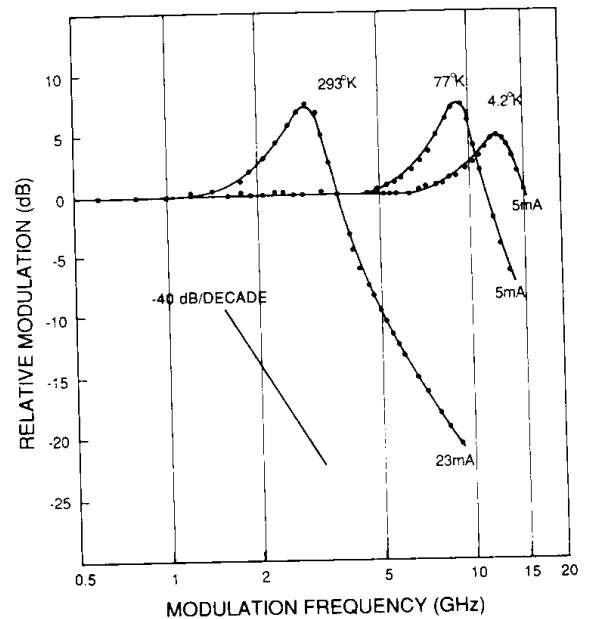

Fig. 7. Active-layer photomixing modulation response data measured at the three operating temperatures. The output power in each is approximately $2 \mathrm{~mW}$ per facet. The resonance in each curve is clearly defined, and the high-frequency rolloff is the theoretically predicted 40 $\mathrm{dB} /$ decade.

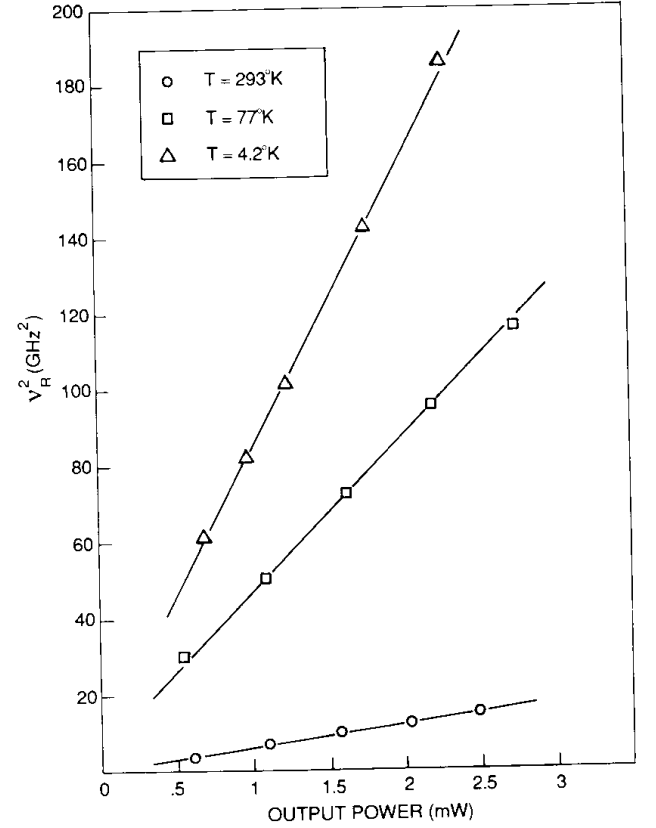

Fig. 8. Square of the relaxation resonance frequency plotted versus the output power per facet. The linear dependence is in agreement with theory. The observed increasing slope with decreasing temperature results from the larger differential gain parameter for low-temperature operation.
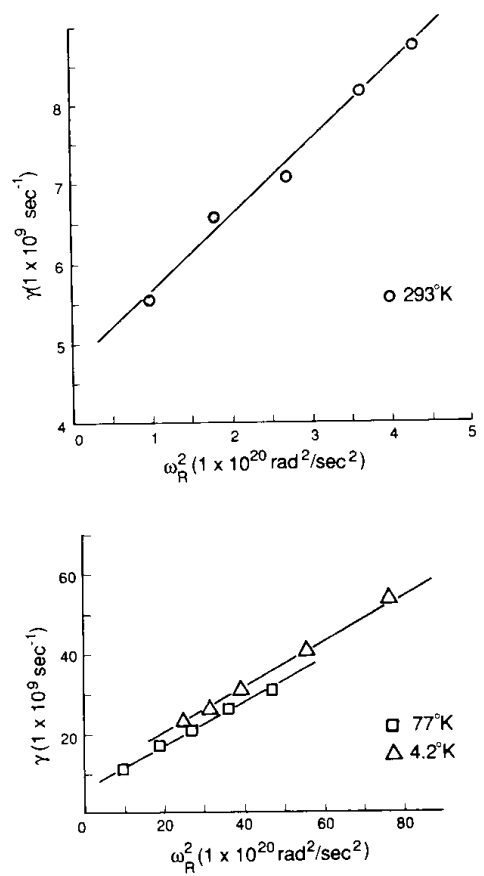

Fig 9. The damping parameter $\gamma$ plotted versus the relaxation resonance frequency for the three operating temperatures. The dependence is linear, as predicted by theory. 
decrease as the temperature is reduced from room temperature to liquid nitrogen temperature. This is consistent with the observed increase in $g_{n}$ and the theoretical result given by $(2 \mathrm{e})$. From liquid nitrogen temperature to liquid helium temperature operation, however, there is no observable change in the slope, despite an additional increase in $g_{n}$. One explanation for this is that $\Gamma\left|g_{p}\right| / g_{n}$ has become much smaller than unity at these temperatures, and the slope as given by $(2 \mathrm{e})$ has saturated at a value equal to $\tau$, the photon lifetime. This seems unlikely, however, since the slope of $5.6 \mathrm{ps}$ at liquid helium temperature is slightly too large for a typical photon lifetime in this structure (we estimate approximately 3 ps based on a cavity length of $250 \mu \mathrm{m}$ and some additional waveguide loss). Therefore, it is more likely that $\left|g_{p}\right|$ is also increasing with decreasing temperature and that it has offset the increase in $g_{n}$ from liquid nitrogen to liquid helium temperature operation. The mechanism for this increase is unknown.

\section{Conclusion}

The overall frequency response of a semiconductor laser to small-signal injection current modulation is the superposition of two independent responses: the parasitic response and the intrinsic response. The parasitic response is determined by extrinsic impedances that tend to shunt current around the laser diode's active layer. The intrinsic response is determined by the basic device physics governing operation of the laser and sets an upper limit on laser modulation speed. Measurement of the intrinsic response is therefore of both fundamental and practical importance. In this paper, a modulation technique we call active-layer photomixing has been presented that can produce optical modulation of a laser diode with near perfect immunity to parasitic effects. The technique produces modulation by photomixing two single-frequency sources in the active layer of a laser diode. This produces modulation of the optical gain and hence modulation of the laser light output. The parasitic-free nature of the technique arises from two effects. First, the modulation is produced where it is needed by selective absorption in the laser active layer; i.e., current transport into the active layer does not play a role in producing the modulation. Second, the modulation, once produced, remains decoupled from the outside world by the extremely small intrinsic impedance of the laser diode. This decoupling was explained by the introduction of an equivalent circuit model for active-layer photomixing.

A TJS laser diode was modulated using the active-layer photomixing technique at temperatures of 293,77 , and $4.2 \mathrm{~K}$. In all cases, the measured response functions were ideal in terms of high-frequency modulation response rolloff, power dependence of the resonant frequency, and dependence of resonance damping on the resonant frequency. This was true even at liquid helium temperature, where the response function was measured to $15 \mathrm{GHz}$ despite an estimated parasitic response corner frequency of only $410 \mathrm{MHz}$. The measured temperature dependence of the relaxation oscillation corner frequency enabled an estimate of the temperature dependence of the differential gain. This, in turn, was correlated with the observed temperature dependence of the slope in the damping data. From this, it appears that the nonlinear gain parameter $g_{p}$ increases with decreasing temperature.

\section{REFERENCES}

[1] K. Y. Lau and A. Yariv, "Ultra-High speed semiconductor lasers," IEEE J. Quantum Electron., vol. QE-21, pp. 121-138, 1985.

[2] M. A. Newkirk and K. J. Vahala, "Parasitic-free measurement of the fundamental frequency response of a semiconductor laser by activelayer photomixing," Appl. Phys. Lett., vol. 52, pp. 770-772, 1988.

[3] K. J. Vahala and M. A. Newkirk, "Measurement of the intrinsic direct modulation response of laser diode by photomixing," presented at the 1988 Conf. Lasers Electro-Opt., Paper MG1.

[4] _ - "Equivalent circuit model for active-layer photomixing: Parasitic-free modulation of semiconductor lasers," Appl. Phys. Lett., vol. 53, pp. 1141-1143, 1988.

[5] C. H. Lange, J. Eom, C. B. Su, J. Schlafer, and R. B. Lauer, "Measurement of the intrinsic frequency response of semiconductor lasers using optical modulation," Electron. Lett., vol. 24, pp. 1131-1132, 1988.

[6] R. S. Tucker and D. J. Pope, "Circuit modeling of the effect of diffusion on damping in a narrow-stripe semiconductor laser." IEEE J. Quantum Electron., vol. QE-19, pp. 1179-1183, 1983.

[7] R. Olshansky, P. Hill, V. Lanzisera, and W. Polwazinik, "Frequency response of $1.3 \mu \mathrm{m}$ InGaAsP high speed semiconductor lasers," IEEE J. Quantum Electron., vol. QE-23, pp. 1410-1418, 1987.

[8] M. Morishita, T. Ohmi, and J. Nishizawa, "Impedance characteristics of double-heterostructure laser diodes," Solid-State Electron., vol. 22, pp. 951-962, 1979.

[9] J. Katz, S. Margalit, C. Harder, D. Wilt, and A. Yariv, "The intrinsic electrical equivalent circuit of a laser diode," IEEE J. Quantum Electron., vol. QE-17, pp. 4-7, 1981.

[10] C. Harder, J. Katz, S. Margalit. J. Schacham, and A. Yariv, "Noise equivalent circuit of a semiconductor laser diode," IEEE J. Quantum Electron., vol. QE-18, p. 333, 1982.

Kerry J. Vahala (S'82-M'84-S'84-M'85), for a photograph and biography, see p. 712 of the April 1989 issue of this Journal.

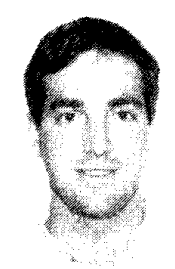

Michael A. Newkirk was born in Palo Alto, CA, on September 5, 1960. He received the B.A. degree in physics from Williams College, Williamstown, MA, in 1983.

At the Fundamental Research Laboratory, GTE Laboratories, Waltham, MA (1984-1986), he was involved in the study of semiconductor lasers and the electronic and nonlinear optical properties of polymeric crystals. Currently, he is a Ph.D. candidate in applied physics under an IBM Doctoral Fellowship at the California Institute of Technology, Pasadena. His research centers on semiconductor laser dynamics and nanometer scale devices. 